\title{
Desobediente: el repositorio digital del Museo Universitario del Chopo
}

Dirección

Clara Martínez Cantón

Gimena del Rio Riande

Francisco Barrón

\section{Secretaría}

Romina De León

Desobediente: the Digital Repository of the Chopo University Museum

Graciela lliana ORDÓÑEZ ALCALÁ

Museo Universitario del Chopo (UNAM)

gracielaordalc@gmail.com

https://orcid.org/0000-0003-3989-1034

Carlos GASCA POSADAS

Museo Universitario del Chopo (UNAM)

carlos.tumbao@gmail.com

https://orcid.org/0000-0001-9647-3600

\section{RESUMEN}

En 2019 se reactivó el proyecto de construcción del Repositorio Digital para la difusión del acervo documental del Museo Universitario del Chopo. Este ha buscado dar acceso al material que conforma los distintos Fondos y Colecciones que son resguardados por el Centro de Información del Museo, a través de los cuales es posible establecer procesos de memoria a la vez que se posibilitan acercamientos a colectivos, proyectos y personas vinculados a prácticas culturales consideradas marginales y underground. Dado que el Museo del Chopo ha tenido un carácter históricamente abierto, en el que se han albergado las disidencias sexuales, artísticas e incluso políticas, estructurar un instrumento de consulta de calidad, constituye un ejercicio democrático y de equidad. En esta nota de investigación se recoge el camino hacia la construcción del repositorio.

\section{PALABRAS CLAVE}

Construcción de comunidad, gestión de proyectos, desarrollo web, modelización, contextualización.

\section{ABSTRACT}

In 2019, the project to build the Digital Repository for the dissemination of the documentary collection of the University Museum of Chopo was reactivated. The project has sought to give access to the material that makes up the different Fonds and Collections in the Museum's Information Center. Through these Fonds it is possible to establish memory processes allowing approaches to groups, projects and people linked to cultural practices considered marginal and underground. The University Museum of Chopo has had a historically open character, in which sexual, artistic, and even political dissidents have been housed, that is why structuring a quality consultation instrument constitutes a democratic and fair exercise. This paper covers the path to building the repository.

\section{KEYWORDS}

Community Building, Project Management, Web Development, Modeling, Contextualization. 


\section{INTRODUCCIÓN}

Cada vez con mayor frecuencia los museos de arte reconocen la necesidad y el valor de la construcción de herramientas digitales que permitan de forma eficiente y estructurada, la consulta en línea de sus colecciones, sean estas artísticas o documentales. En México, sin embargo las iniciativas siguen sin ser generalizadas y los caminos permanecen agrestes. En ese ambiente, hemos querido compartir el proyecto de construcción del repositorio digital para el acervo documental del Museo Universitario del Chopo, el Archivo Desobediente'. Para nada nuestra intención es establecer mapas de ruta o soluciones definitivas. Se trata más bien de un proceso de autorreflexión que busca evidenciar lo contingente y problemático. Vamos constantemente de lo pragmático y acaso anecdótico a la reflexión y aprendizaje metodológico y teórico, pues ese ir y venir ha jugado un papel importante en el desarrollo del proyecto. Retomando las reflexiones en torno al archivo y los procesos de investigación de Mario Rufer (2016), proponemos dar cuenta de esos procesos que se suelen neutralizar, para desmontarlos y repotenciarlos. El objetivo se centra en presentar el proceso seguido en la construcción del Archivo Desobediente, con las nociones y soluciones que le han dado forma, como una posible contribución en el ámbito de la construcción de herramientas digitales que colaboren en la democratización del conocimiento y la visibilización de memorias diversas y subrepresentadas.

Como veremos, el Museo del Chopo tiene una historia peculiar con respecto a su relación con lo digital, a la que hemos tratado de sintonizarnos no sin cierto posicionamiento crítico. Acuden a éste la cultura DIY (do it yourself), el software de código abierto, los modelos de metadatos, el control de vocabularios y las culturas underground.

\section{ENTRE LO EFÍMERO Y LA ESTRUCTURA. EL MUSEO DEL CHOPO COMO PUNTO DE PARTIDA}

Hoy es quizá de lo más frecuente pensar en el museo de arte como un espacio de libertad, experimentación y encuentro, en el que suceden cosas de la más diversa índole y pertenencia disciplinar, en atención a los diversos públicos que se dan cita en ellos. Sin embargo, en 1975, cuando se fundó el Museo Universitario del Chopo de la UNAM (MUCH) (Museo Universitario del Chopo, 1975), pensar en un espacio reconocido, identificado y reivindicado como museístico, pero no necesariamente centrado en una colección artística, ni en la contemplación de objetos y visualizado mucho más allá de las exposiciones, no era un ejercicio habitual. Desde luego, sería equivocado considerar que el proyecto del Museo del Chopo naciera como algo acabado, pero bien puede decirse que se tuvo claro desde el principio que sería un espacio distinto, capacitado para convocar manifestaciones jóvenes, alternas, emergentes y no necesariamente ubicadas en los polos tradicionales de cultura de la Ciudad Autónoma de México. Así, esa apertura lo llevó muy pronto a vincularse con las dinámicas de su entorno inmediato y a ser tomado por las disidencias sexuales, musicales,

\footnotetext{
1 Accesible desde: $\underline{\text { http://archivodesobediente.chopo.unam.mx. }}$
} 
artísticas, políticas y, en colaboración con ellas, gestar actividades que incluyeron conciertos, conferencias, foros, seminarios y talleres. El Chopo tomó de esa forma el carácter de lugar de diálogo, mediación, inclusión, pluralidad y cercanía comunitaria con que se le identifica y que es ya recurrente en la definición de los museos.

Es posible mirar los diversos tipos de acercamientos que se fueron construyendo, así como lo que podría significar ese carácter de lugar de disidencias, si se tienen en cuenta algunos de los eventos y las actividades que se realizaron en el museo desde sus primeros años. Encontramos por ejemplo el Primer concurso de composición El rock en el Chopo de 1979 y el Primer Tianguis de la Música de 1980 (Museo Universitario del Chopo, 1980) (que luego se convertiría en el Tianguis Cultural del Chopo) como confluencias con el rock, como invitación a la creatividad juvenil y a la circulación libre de la cultura y la información en forma de trueque, en respuesta a la escasez de espacios para adquirir y compartir. Está también la Semana cultural lésbico-gay (a partir de 1987) (2019) como un lugar de visibilidad, reivindicación y discusión de temas urgentes marginados de espacios institucionales. Igualmente significativas como respuesta a la marginación y el rechazo, las Jornadas del movimiento punk (1988) implicaron el justo reconocimiento a un movimiento radical y significaron un diálogo más profundo con el underground. Las Jornadas de lucha contra el SIDA (a partir de 1990) se convirtieron en un foro multidisciplinario de información, reflexión y activismo que pugnó contra la estigmatización de las personas que viven con $\mathrm{VIH}$. Importantes son de igual manera, los diferentes actos de solidaridad con países centroamericanos que tuvieron lugar, entre los que podría ubicarse la Medalla Roque Dalton (1985-2000), cuya sede siempre fue el Museo del Chopo y que se otorgaba a personas $u$ organizaciones destacadas en el activismo y los derechos humanos ${ }^{2}$.

Este recorrido, para nada exhaustivo, nos permite más bien un vistazo a las posibilidades que el museo construyó o a las que se construyeron en él. Hablamos más que de objetos, de relaciones entre personas, de redes para compartir conocimientos e iniciativas, propiciar intercambios capaces de responder a la marginación, prácticas casi siempre colectivas que reclamaban un espacio negado, diálogos solidarios que buscaban acompañar luchas diversas. Ante ese panorama, lo que reconocemos es un rasgo experiencial que produjo a su vez, porque lo hizo a pesar de no ser su interés primordial, documentos más bien efímeros tanto en su materialidad como en su intención. Estos documentos se produjeron alejados de una lógica de archivo y de prácticas de archivado, enfocados más bien en convocar y replicar mensajes inmediatos.

Sin embargo, conforme el Museo del Chopo creció y sus tareas, áreas y personal se ampliaron, se añadieron igualmente prácticas que estructuraban los resultados materiales de las actividades realizadas. De tal suerte que se generaron formas, posiblemente orgánicas pero sostenidas, concebidas ya para dejar memoria de lo realizado. Encontramos así las publicaciones a partir

\footnotetext{
2 La Medalla se entregaba por el Consejo de Cooperación con la Cultura y la Ciencia en El Salvador, una asociación civil. Algunos de los múltiples galardonados fueron Samuel Ruiz, Digna Ochoa y Carlos Montemayor.
} 
de las exposiciones, las compilaciones de notas de prensa en donde se hacía referencia a los contenidos del Museo, así como los materiales de registro fotográfico y audiovisual. Con ello podríamos ubicar una tensión fundante en la historia del Museo, entre las prácticas abiertas y efímeras y el interés por estructurar, organizar y resguardar con el ojo puesto en el futuro, entre la diversidad y convivialidad a la que convocaba y los requerimientos y obligaciones institucionales a los que se adscribía como museo perteneciente a la UNAM.

Con eso en mente, queremos mencionar dos momentos importantes en la propia historia del Museo en los que esa tensión se revisó y reacomodó, en los que sobre todo se pusieron en juego elementos para repensar el pasado del Chopo y generar herramientas de vinculación con él. Hablamos primero del desarrollo del proyecto de la Mediateca del Museo, vinculado con la reapertura del mismo tras su remodelación en 2010. Ese proyecto de gran envergadura buscó establecer las condiciones para crear una plataforma de acceso al archivo a partir de un trabajo de catalogación y la digitalización masiva, la creación de la infraestructura tecnológica e institucional, a través de servidores propios y la contratación de un software propietario, por un lado, y a la reestructuración de las áreas dedicadas a la documentación y la creación de espacios de consulta y almacenamiento, por el otro.

Lo que hoy es el Centro de Información y Mediateca (CIM), surgió entonces, como el área del Museo encargada de la gestión, resguardo, acceso y difusión del acervo documental, con un énfasis en el material visual y gráfico resultado de las actividades culturales realizadas: ephemera, catálogos y folletos de exposiciones, material fotográfico y audiovisual de registro, entre otros. El eje del desarrollo del acervo se orientó principalmente a los materiales capaces de dar cuenta en imágenes del recorrido y los agentes participantes en el recorrido que el Museo había tenido desde su fundación.

El proyecto de la Mediateca alcanzó varios de sus objetivos, entre ellos el crear bases de datos que permitían la búsqueda y recuperación de la información de los usuarios dentro de las instalaciones del CIM, así como la digitalización de miles de documentos que conforman el acervo. Sin embargo, el proyecto tuvo también muchas limitaciones y problemas. Como parte de éstas pueden mencionarse las limitantes en la catalogación y en el modelo seleccionado para ella o, más bien, los modelos seleccionados (pues se trabajaba con distintas bases de datos, cada una bajo un modelo de datos no estandarizado). Por otra parte, problemas con el software y lo restringido de las interfaces de usuario fueron parte de esas problemáticas.

El otro momento es el abierto a partir de 2012 con la llegada a la Dirección del Museo de José Luis Paredes Pacho, cuyo programa incluyó una revisión y relectura de la historia del Museo y de los diferentes actores y comunidades que en las décadas previas habían sido parte de él (Museo Universitario del Chopo, 2019). Con ese objetivo, hubo un interés por reactivar y formalizar esa cercanía, convertida esta vez en estrategias de resguardo y difusión de las memorias del underground de los años 80 y 90 y de la escena cultural y artística alternativa en México. Surgieron de esa manera las Colecciones Heterodoxias, que han significado la creación de colecciones digitales, en un esfuerzo por reducir en alguna manera su vulnerabilidad y aumentar su difusión. Algo 
similar ha ocurrido con el proyecto Fanzinoteca, enfocado en las prácticas de autopublicación, el cual, en colaboración con coleccionistas y productores, ha conformado un archivo físico y digital, que al mismo tiempo que recopila y abriga parte de dichas prácticas de autogestión, buscando reconocer a las comunidades que se vinculan a partir de ellas.

Estos elementos configuran el entorno desde el cual partimos y en el que se inscribió el proyecto de repositorio digital. Debíamos reconocer esa trayectoria y proponer una herramienta que respetara la particularidad de la historia del Museo Universitario del Chopo y que fuera lo suficientemente abierta como para dar cabida a la documentación con las características antes enunciadas. Requeríamos atender, y no eliminar esa tensión entre lo efímero y la estructura. Era necesario, además, poner en su justo valor los proyectos previos y tomar conciencia que nuestro punto de partida no era cero. Este es entonces, el recorrido que planteamos, las nociones que nos acompañaron y las soluciones propuestas, en un ejercicio de construcción y aprendizaje continuos.

\section{ADMINISTRAR COLECCIONES DESDE El SOFTWARE DE CÓdIGO ABIERTO Y CATALOGAR OBJETOS CULTURALES EN SENTIDO AMPLIO}

En 2019 se reactivó el proyecto para la construcción de una herramienta digital de difusión y consulta para el acervo documental del Museo Universitario del Chopo. Dados los antecedentes antes explicados, se buscó aprovechar los recursos disponibles y atajar los desaciertos de los proyectos desarrollados previamente. Era fundamental que se tratara de una plataforma sobre la que se tuviera poder de decisión, en la que la integridad de datos y objetos digitales estuviera garantizada y que, desde luego, tuviera que ver con el recorrido y perfil del Museo e incorporara, en sus preceptos y capacidades, los programas que se habían integrado a partir de 2012. La idea básica era contar con una herramienta de catalogación y gestión que permitiera el uso de estándares de metadatos y ofreciera una salida pública atractiva y funcional.

Partimos de la convicción de trabajar con software libre o de código abierto, como un posicionamiento que construía vínculos con las formas de producción y circulación de parte importante del acervo del Museo, y con el fin pragmático de establecer dinámicas de trabajo igualmente abiertas. Hay en el software de código abierto principios cooperativos basados en internet que representan un tipo de bien común (Schweik, 2016) en el que hemos querido participar. Si en los criterios para definir un software de código abierto se encuentran la redistribución libre, el código fuente legible y las obras derivadas autorizadas (Open Source Iniciative, 2007), en las comunidades de usuarios hay una disposición sostenida a establecer redes de conocimiento y apoyo entre pares que terminan por cuidar y acompañar los proyectos. Traer esas dinámicas a las lógicas institucionales, abre algunas grietas por las que se fractura un poco su rigidez y disciplina, al mismo tiempo que introduce la posibilidad de cooperación, en la que la institución pública puede, por ejemplo, participar en el desarrollo de infraestructura.

Luego de una investigación que se centró en la revisión general de distintas opciones de software libre o de código abierto, encontramos en Collective Access (CA) la solución más adecuada. Este un software de código abierto diseñado para administrar y publicar colecciones de archi- 
vos y museos ${ }^{3}$. El sistema tiene soporte para una amplia variedad de estándares de metadatos y ofrece una gran cantidad de funciones que permiten administrar, validar, relacionar, organizar, importar/exportar y mostrar datos y representaciones de objetos. Se compone de dos módulos independientes, uno encargado de la gestión y la catalogación, Providence, y el otro, Pawtucket, dedicado a la difusión y salida pública del archivo (Whirl-i-Gig, 2019). Este último da la posibilidad de presentar galerías que pueden funcionar a manera de exposiciones virtuales.

Esta gama de posibilidades hace de CA una herramienta altamente adaptable pero no necesariamente cristalina. En ese sentido, su uso implica una serie de retos y aprendizajes no siempre fáciles de sortear y adquirir. Es aquí donde, como en todo el software de código abierto, adquiere mayor importancia la comunidad de usuarios y las redes que se puedan tejer entre proyectos con características e inquietudes comunes. En el caso de esta aplicación, existe una comunidad muy importante, que sin embargo proviene en su mayoría, de países de habla inglesa, con condiciones muy alejadas a las nuestras. Recientemente el uso de este software comienza a darse en México y otros países de América Latina como Argentina y Chile, y se abre con ello, la posibilidad de dialogar ahora sí en escenarios semejantes.

En cuanto al modelo de datos, tras un análisis y valoración de diferentes estándares de metadatos, las necesidades de los fondos y colecciones que resguarda el $\mathrm{CIM}^{4}$ del Museo, y su compatibilidad con Collective Access, se optó por la utilización de CDWA-Lite por su amplitud y versatilidad, en su disposición para describir obras de arte, materiales culturales, ítems y conjuntos de ítems (colecciones). CDWA-Lite permite cubrir de buena manera el rango de tipologías documentales, orígenes y formas de uso de los materiales que conforman el acervo documental del Museo del Chopo, se basa en Cataloging Cultural Objects (CCO), que es un estándar de catalogación de contenido para objetos culturales. En su definición estricta, este software no se entiende como un modelo de datos, sino como un conjunto de pautas para las buenas prácticas de catalogación y descripción de objetos culturales y de arte (J. Paul Getty Trust, 2019); una suerte de marco conceptual para estructurar la información, establecer relaciones y jerarquías entre los elementos. Asimismo, por su parte, esta aplicación es la expresión técnica, un esquema XML que posibilita la codificación de datos en una base (Boughida, 2005). En su conjunto, admite mirar al acervo del Museo con una traza amplia, cercana a su singularidad, en la medida que logra trabajar con objetos culturales y con obras artísticas, un cruce común en la institución. Igualmente, ese modelo y esquema, viabiliza la inclusión futura de la colección artística con la que cuenta El Chopo.

\footnotetext{
${ }^{3}$ Collective Access requiere lo que se reconoce como un entorno AMP (Apache, MySQL y PHP) (Alcaraz Martínez, 2014). Valga recordar aquí lo que consigna Schweik (2016): "A pesar de que se den o se vayan a dar fracasos en este ámbito cooperativo, son más numerosas las historias de éxito (medidas en términos de crecimiento del uso del software o de cuota de mercado), con casos prominentes como el sistema operativo Linux, el Servidor Apache, Php (un lenguaje de secuencia de comandos de código abierto), MySQL (una base de datos relacional de software libre de código abierto) [...]" (p. 297).

4 El Centro de Información y Mediateca, es el área del Museo que se encarga actualmente de la del proyecto del repositorio digital.
} 


\subsection{Las Colecciones Heterodoxias y Fanzinoteca de todxs en el repositorio}

En general, el acervo que resguarda el CIM, y en particular, las colecciones que actualmente han sido ingestadas, en lo que decidimos nombrar como Desobediente, Archivo Digital del Museo Universitario del Chopo, tienen un carácter underground marcado por la tipología de los objetos que las conforman, así como por los rasgos de sus creadores.

Hasta el momento, las colecciones que se encuentran ingestadas en el repositorio son:

- Colección Mauricio Bares. Es un repertorio pequeño, compuesto por nueve revistas editadas independientemente por Mauricio Bares durante la década de los noventa. Prontamente dentro del proyecto, se convirtió en una de nuestras favoritas, no sólo por sus características de género y forma, sino también porque se volvió una herramienta que nos permitió conocer y experimentar con el CDWA-lite y su aplicación en Collective Access.

- Colección Gráfica Mongo, concentra parte de la producción gráfica de Ramón Sánchez Lira "Mongo", creador de la imagen de La Última Carcajada de la Cumbancha y el Disco-Bar Nueve durante la década de los ochenta y principios de los noventa. Esta colección consta de 214 ítems cuyas tipologías documentales abarcan volantes, carteles, folletos, invitaciones, etc., como promocionales de los eventos a realizarse en estos espacios culturales nocturnos de la Ciudad de México.

- Colección Carlos Alvarado/Tianguis Cultural del Chopo, es una colección de 121 ítems que ofrece un testimonio de la vida del Tianguis Cultural del Chopo, su historia y relación con el propio Museo. Fue compilada por Carlos Alvarado y reúne recortes de revistas y periódicos, así como volantes, carteles, invitaciones, folletos que fueron producidos y difundidos en el tianguis.

- Fanzinoteca de Todxs merece una mención aparte. Se compone precisamente por fanzines y publicaciones independientes, que responden a una dinámica propia, que quieren escapar de las convenciones editoriales de libros y revistas, por lo que intentar describirlas en esos términos no resulta pertinente. En este contexto, los fanzines constituyen su propia unidad de información, la fanzinoteca, con sus propias dinámicas de organización, descripción y difusión generadas a partir de los ideales de la cultura hágalo usted mismo (DIY)5. Puede entenderse como un medio de difusión no mercantil, al igual que las bibliotecas, de publicaciones independientes que paradójicamente no han tenido cabida en la mayoría de las bibliotecas y archivos.

\footnotetext{
5 Explica un investigador: "Surgido de la cultura underground, el Do It Yourself (DIY) ○ Hazlo tú mismo se convirtió en una forma de creación y distribución cultural que tuvo su mayor repercusión en los albores del movimiento punk, convirtiéndose a partir de ese momento en una alternativa a la creación mainstream [...]" (Gallego Pérez, 2009, p. 79).
} 
En cuanto a sus prácticas de catalogación (cuando las tienen), no necesariamente son llevadas a cabo por bibliotecarios o archivistas "profesionales", y generalmente suele priorizar la difusión y el uso de los materiales a su preservación.

\subsection{Catalogación y control de vocabularios}

Como se expuso más arriba, alineado con el contexto en el que nació el MUCH (no podía ser de otra forma), Desobediente, archivo digital del Museo Universitario del Chopo se pensó como un espacio de disidencias, de creación y circulación libre de la información. Algunos objetivos que se plantearon fueron el de representar la diversidad de la información resguardada en la CIM; a partir de distintas dinámicas, generar un espacio para compartir conocimientos y abrir un diálogo en un ambiente digital; establecer un instrumento de consulta de calidad que visibilizara las creaciones, experiencias y conocimientos de grupos y personas que, de alguna forma, no han estado representados en galerías, bibliotecas, archivos o museos. Estos objetivos, en su aplicación, han tenido que ser armonizados con los procesos técnicos de organización y normalización de datos para su recuperación en un sistema de información, pero también nos han llevado a reflexionar de forma constante sobre las prácticas de organizar, clasificar, describir y difundir información y temas considerados marginales como actos disidentes y empoderantes en sí mismos.

Para llegar al punto actual, la primera versión del repositorio ha caminado un largo trayecto que involucra los esfuerzos y conocimientos de distintos actores que han participado, ya sea donando sus materiales, catalogando o digitalizando. En este sentido, el proyecto ha sido colaborativo y en diálogo con los procesos desarrollados por otros que nos antecedieron. Para su construcción reutilizamos las descripciones que se hicieron en otro momento en el CIM y con ellas el conocimiento e investigación de sus catalogadores, un ejemplo de esto puede encontrarse en la Colección Gráfico Mongo, cuyas notas descriptivas son ricas en detalles sobre personas y lugares vinculados con el objeto, además, cuenta con un análisis de la composición en los carteles, volantes y folletos que comprenden este grupo documental. De este modo, para la construcción de Desobediente, reutilizar las bases de datos nos permitió optimizar los recursos y esfuerzos. El proceso de reutilización de los datos consistió en la siguiente metodología:

- Análisis de la base de datos original6, poniendo especial atención en las tipologías documentales que describen sus registros, así como los atributos y características de los objetos descritos.

- Mapeo conceptual entre la estructura de la base de datos original y las categorías y subcategorías de Categories for the Description of Works of Art (CDWA), en este documento se asientan las condiciones del mapeo, las cuales indican cómo estructurar la base de datos y normalizar.

- Diseño y estructura de base de datos a partir de CDWA.

\footnotetext{
6 Por bases de datos originales nos referimos a aquellas creadas entre los años 2013 y 2015 durante el proyecto de catalogación de estos materiales.
} 
- Aplicación de vocabularios controlados, ajuste de la sintaxis en campos de texto libre.

Debido al contexto en el que se desarrollaron los grupos y personas que crearon estos objetos, marginal a los circuitos tradicionales de creación y difusión, y pertenecientes a una escena local underground de la Ciudad de México, ha sido necesario elaborar nuestro propio control de autoridades para entidades personales y corporativas.

Hasta el momento, los registros de autoridades elaborados para entidades personales y corporativas son sencillos, recogen un identificador y proporcionan para las denominaciones principales: el nombre de pila, segundo nombre, apellido o nombre de la organización (este último para el caso de entidades corporativas, así como el nombre para visualización). En pocos casos se han registrado denominaciones alternativas y datos biográficos, debido a que existen muy pocas referencias publicadas sobre estas entidades. La investigación ha sido complicada y nos ha llevado a fuentes validadoras informales como las fuentes originales o cuentas en redes sociales. Se ha pensado, para una siguiente etapa, impulsar proyectos que involucren a las personas y grupos responsables de la creación de estos materiales en el registro de sus propias autoridades como un ejercicio de autodeterminación.

Para las autoridades de lugar, se validaron los términos con el Tesauro de Nombres Geográficos (TGN) y los catálogos del Instituto Nacional de Estadística y Geografía, INEGI. Igualmente, Collective Access cuenta con una función de georreferencia que se encuentra enlazada con Google Maps, sin embargo, hasta el momento no hemos utilizado esta aplicación.

Para el campo Tipo de obra/objeto, pudimos utilizar para validar los términos, en gran medida, el Tesauro de Arte y Arquitectura (AAT), sobre todo para las colecciones Gráfica Mongo, Carlos Alvarado/Tianguis Cultural del Chopo y Mauricio Bares. Por su parte, los objetos de la colección Fanzinoteca de Todxs no corrieron con tanta suerte. Como se comentó previamente, esta última constituye una unidad de información propia que no ha sido estudiada suficientemente, si bien existe el término validado por AAT de fanzine, creemos que en su contexto le dice muy poco al usuario. Fue entonces que comenzamos una investigación (que aún no concluye) de términos específicos relacionados jerárquicamente con el término general de fanzine; término más específico para designar el tipo de obra/objeto en las colecciones de la misma, que permite identificar de forma más específica el contenido, ofrecer un punto de acceso más especializado o, al menos planteado desde el ámbito de esta, y visibilizar la diversidad de género y forma de este tipo de publicaciones independientes. Las fuentes en las que se validaron principalmente estos términos fueron Zine Subject Thesaurus de la Anchor Archive Zine Library ${ }^{7}$, la lista de géneros de Bernard Zine Library ${ }^{8}$, así como el listado denominado Contenidos en La Fanzinoteca9.

Por otro lado, crear vocabularios controlados adecuados a las necesidades de descripción

\footnotetext{
7 Accesible desde: https://anchorarchive.org/subject-thesaurus-pdf.

8 Accesible desde: https://zines.barnard.edu/zine-genres.

9 Accesible desde: http://fondo.fanzinoteca.net/.
} 
de los objetos que se encuentran en el repositorio ha significado ventajas en términos de catalogación, pero también inconvenientes debido a que en la instalación de Collective Access está dado por default el Tesauro de Arte y Arquitectura. Si se desea cargar un vocabulario o listado propio se debe hacer a partir de mecanismos de ingesta manuales o automatizados, estos procedimientos significan investigación y pruebas que se han incluido en el proceso de aprendizaje que hemos tenido al trabajar con Collective Access. Al crear nuestros propios vocabularios controlados (un poco quizás, bajo la consigna hágalo usted mismo", en combinación con fuentes estandarizadas, hemos intentado establecer un diálogo a través de un vocabulario común entre el mundo underground, de naturaleza contestataria, y el mundo de la catalogación y los lenguajes de recuperación implícitos en los repositorios. Dicho diálogo, como tal, está abierto a la réplica, por lo que es primordial para nosotros generar una política de catalogación abierta que respete tanto la naturaleza de los objetos como de sus creadores y, al mismo tiempo, ofrezca información relevante y puntos de acceso útiles al usuario. Para tal efecto, hemos insertado, en el campo Derechos de la obra el siguiente texto:

En sintonía con el carácter abierto y libre con que se identifica el fanzine, compartimos esto con la intención de ofrecer una ventana distinta de difusión. Si participaste de alguna manera en la publicación y consideras que no recibes el reconocimiento adecuado o estás en desacuerdo con que tu publicación aparezca aquí, escríbenos. Si, por otra parte, te interesa la reproducción de este material, ponte en contacto con los creadores.

Construir Desobediente Archivo Digital del Museo Universitario del Chopo ha sido una oportunidad de visibilizar, en sus propios términos, las experiencias y conocimientos de comunidades que han sido marginadas por distintas razones (políticas, sociales, culturales, ideológicas, económicas) y de repensar las prácticas de catalogación y de autodescripción como una forma de empoderamiento (Thompson, 2016).

\section{COMPARTIR Y COLABORAR: LO QUE PODEMOS CONSTRUIR EN ADELANTE}

Fue así como llegamos a Desobediente, en ese transcurrir entre investigación y acción, nos hizo comprender el carácter disidente de gran parte de lo contenido en el acervo documental del Museo del Chopo, pero también registramos la existencia de lógicas de orden y control. Nos mantenemos en esa tensión en la que hemos insistido, pero advertimos la disciplina que implican los sistemas de archivado y la forma en que son parte por cómo constriñen y burocratizan la experiencia de una cultura de la obediencia (Rufer, 2016). Hemos querido trabajar en otra dirección, y en todo nuestro proceso, con sus contradicciones, intentamos reconocer la desobediencia, en lo artístico y en lo archivístico. Ante esta posible paradoja, proponemos con Marina Garcés a partir de F. Deligny, el recurrir a la imagen de la Balsa, troncos atados entre ellos de tal forma que quedan holgados, en la que tan importante son los vínculos y la atadura, como la distancia: "Nuestra libertad relativa depende de esta estructura. Libre no es quien se lanza a mar abierto sino quien es capaz de elaborar el dispositivo y las relaciones necesarias para dejar la orilla sin ahogarse" (Garcés, 2014 , p. 20). Nos movemos entonces "entre el adentro y el afuera, la institución y los movimientos, la espontaneidad y la organización, la construcción y la destrucción, la estabilidad y la movilidad, 
la solidaridad y el antagonismo" (p. 20).

Este, sin duda, no es el fin del trayecto, pues nos falta aún bastante por aprender sobre Collective Access. Las descripciones de las colecciones creadas hasta ahora pueden detallarse mucho más y queda también trabajo pendiente en materia de vocabularios controlados y de explorar las relaciones entre entidades. También, entre estos objetivos, están la ingesta del resto de las Colecciones Heterodoxias, otras colecciones de fanzine y el Fondo Histórico del Museo. De capital importancia es el activar repositorio como un espacio de apropiación para la comunidad de usuarios y creadores a partir de dinámicas de desarrollo de colecciones y autoarchivo. Collective Access tiene algunas aplicaciones en este sentido que buscamos explorar.

Otra cuestión significativa es, ya se han dado algunos pasos en este sentido, el establecer redes para compartir experiencias del uso y aplicación de CDWA-Lite y Collective Access, que nos permitan potenciar el uso de estas herramientas como comunidad a nivel local y en la región de Latinoamérica. Sin duda, la construcción de Desobediente no ha sido un proceso lineal: hemos avanzado, pero también hemos tenido que dejar reposar, para volver a después con una mirada nueva e ímpetu. Pero este esbozo aquí planteado nos pone en perspectiva la parte del trayecto ya realizado ${ }^{10}$.

\section{REFERENCIAS BIBLIOGRÁFICAS}

Alcaraz Martínez, R. (2014). Collective Access, un sistema de gestión y difusión de colecciones de museos, archivos y bibliotecas. En BiD: textos universitaris de biblioteconomia i documentació, 33. http://dx.doi.org/10.1344/BiD2014.33.23

Boughida, K. B. (2005). CDWA Lite for Cataloguing Cultural Objects (CCO): A New XML Schema for the Cultural Heritage Community. En Humanities, Computers and Cultural Heritage, Proceedings of the XVI international conference of the Association for History and Computing. Royal Netherlands Academy of Arts and Sciences. https://dans.knaw.nl/nl/over/organisatie -beleid/publicaties/DANShumanitiescomputersandculturalheritageUK.pdf\#page $=49$

Flinn, A. (201 1). Archival Activism: Independent and Community-led Archives, Radical Public History and the Heritage Professions. InterActions: UCLA Journal of Education and Informations Studies, 7(2). https://escholarship.org/uc/item/9pt2490x

Freedman, J. (2009). Grrrl Zines in the Library. Comparative Perspective Symposium Feminist Zines, Sings, 35(1). https://doi.org/10.1086/599266

Freedman, J. (2014). Punk As a Zine Library. En Maximum Rocknroll. https://doi.org/10.7916/ $\underline{D 82 Z 13 M 0}$

Gallego Pérez, J. (2009). Do It Yourself, cultura y tecnología. Revista Icono, 14, 278-291. https:// doi.org/10.7195/ri14.v7i2.327

Garcés, M. (2014). Común (sin ismo). Pensaré cartoneras.

10 El repositorio del Museo Universitario del Chopo es público y puede consultarse desde la siguiente dirección: http://archivodesobediente.chopo.unam.mx/. 
J. Paul Getty Trust \& College Art Association. (2019). Categories for the Description of Works of Art https://www.getty.edu/research/publications/electronic publications/cdwa/definitions.pdf

Museo Universitario del Chopo. (1975). Museo Universitario del Chopo [cartel]. http:// archivodesobediente.chopo.unam.mx/index.php/Detail/objects/4964

Museo Universitario del Chopo. (1980). Tianguis de la música [cartel]. http:// archivodesobediente.chopo.unam.mx/index.php/Detail/objects/507

Museo Universitario del Chopo. (1988). Jornadas del movimiento punk [cartel]. http:// archivodesobediente.chopo.unam.mx/index.php/Detail/objects/5012

Museo Universitario del Chopo. (2010). Proyecto ejecutivo de Mediateca [documento no publicado].

Open Source Initiative (2020). The Open Source Definition. https://opensource.org/docs/osd

Rufer, M. (2016). El archivo: de la metáfora extractiva a la ruptura poscolonial. En F. Gorbach \& M. Rufer (Coords.). (In)disciplinar la investigación:archivo, trabajo de campo y escritura (pp. 160-186). Siglo XXI Editores, Universidad Autónoma Metropolitana.

Schweik, C. (2016). El software libre de código abierto como marco para el establecimiento de bienes en la ciencia. En Charlotte Hess, Charlotte y Ostrom, Elinor (Ed). Los bienes comunes del conocimiento (pp. 295-327). Instituto de Altos Estudios Nacionales, Traficantes de sueños. Thompson, K. (julio, 2016). More Than a Name: A Content Analysis of Name Authority Records for Authors Who Self-Identify as Trans. En Library Resources \& Technical Services, 60(3), 140155. https://journals.ala.org/index.php/Irts/article/view/6036

Whirl-i-Gig. (2019). Collective Access. Features. https://www.collectiveaccess.org/features 